\title{
Outcome of Primary Repair of Severed (Partial/ Complete) Tendoachilles
}

\author{
1. Dr. Farhan Imtiaz Chowdhury, \\ MBBS, FCPS (Surgery), \\ Registrar Surgery \\ Rajshahi Medical College Hospital \\ Rajshshi.
}

\author{
Dr. Md. Musab Khalil, \\ MRCP(UK), MD (Gastroenterology), \\ Assistant Registrar, \\ Sheikh Russel National Gastroliver Institue and Hospital, \\ Mohakhali, Dhaka
}

\author{
2. Dr. Mir Rasekh Alam Ovi, MBBS, \\ FCPS (Surgery), FMAS \\ Consultatnt, Surgery \\ General Hospital, Narayangonj, \\ Dhaka.
}

\author{
4. Dr. Shamima Nasrin, \\ MBBS, FCPS (Surgery), FMAS \\ Assistant Professor Surgery \\ Ad-din Women's Medical College \& Hospital, \\ Dhaka, Bangladesh.
}

\begin{abstract}
:-
$>$ Introduction:

Rupture of the Achilles tendon was reportedly first described by Ambroise Pare in $\mathbf{1 5 7 5}$ and was reported in the literature in 1633. After the 1920s the use of operative treatment increased, primarily because of reports by Abrahamsen in 1923 and Quenu and Stoianovitch in 1929 who advocated surgical repair, based on accumulated cases from the literature. In this series we used 5 parameters according to Juhana Leppilahti Modified scoring system to assess the final outcome of the patient functionally. These were stand on affected tip toe unsupported, range of motion of affected side ankle, power of planter flexion, calf muscle wasting and complications. These parameters were also used in various study described in various literature. So, early repair of open Tendo Achilles injury provided an effective outcome, which was comparable with the result described in others.
\end{abstract}

\section{Methods:}

The study was designed as a prospective cohort study conducted at Rajshahi Medical College Hospital from January 2017 to December 2017. Study was carried out on 50 patients hospital admitted with ruptured tendoachillis, irrespective of age and sex. Modified Kessler was the procedure as operative treatment for their disease. Outpatient follow-up was undertaken.

\section{Result:}

In our study among 50 patients $38(76 \%)$ were male and $12(24 \%)$ were female. Mean age was 30.02 years with standard deviation of \pm 7.7 . Most of the cases were operated within 6 hours of injury. After final toileting wound swab was sent for culture sensitivity and found in $60 \%$ cases $E$. Coli, 6\% Pseudomonas, 4\% Klebshiela and in $30 \%$ cases no growth. Mean delay of repair was 6 hours 7 minutes, with standard deviation $\pm \mathbf{2 . 3 6 2}$ minutes. In our study median level of cut was $3.0 \mathrm{~cm}$ proximal to the insertion of Tendo Achilles and where interquartile range was 2.5 to 3.5 cm. Mean follow up period was 9 months with standard deviation was 2.37. Ankle motion were normal in 35 cases $(70 \%)$ and changes of motion were up to 6-10 degree in $13(26 \%)$ patients in this series, which was very much acceptable in comparison to above studies. Only 2(4\%) patients had more than 10 degree motion restricted. Common complications were superficial skin infection 4 cases $(37 \%)$, mild swelling 4 cases (36\%), ugly scar 2 cases $(18 \%)$ and wound gap 1 cases $(9 \%)$. In our study no major complication occurred but minor complications like superficial skin infection, delayed wound healing occurred which were successfully managed with an oral antibiotic regimen with no long term sequelae. In this series we assessed fifty acute open Tendo Achilles injured patients after 16 weeks of treatment. Among them 90\%(45) can stand on affected tip toes unsupported, around 4 cases $(8 \%)$ cannot stand on tip toes of affected foot can't support but able to stand on affected foot with unsupported. Around 2\% (1 case) failed to stand on affected foot without support due to severe infection and wound gap. The final result was as followed excellent in 28 cases $(\mathbf{5 6 \%})$, good in 16 cases (32\%), fair in 4 cases $(8 \%)$, poor in 2 cases $(4 \%)$. In this study it was found that $85 \%$ satisfactory result among 50 patients of acute open tendo Achilles injured patients by immediate repair. If this procedure put in total population then satisfactory result was found in following confidence interval (at 5\% level of significance). According to Juhana Leppilahti Modified Score at $\mathbf{9 5 \%}$ Confidence Interval satisfactory outcome is $78.99 \%$ to $97.00 \%$, which was comparable with the result described in others.

\section{Conclusion:}

Early repair of open Tendo Achilles complete or partial provided an effective outcome, which was comparable with the result described in others.

Keywords:- Tendoachilles, Outcome, Primary Repair. 


\section{INTRODUCTION}

Rupture of the Achilles tendon was reportedly first described by Ambroise Pare in 1575 and was reported in the literature in 1633.1 After the 1920s the use of operative treatment increased, primarily because of reports by Abrahamsen2; Quenu and Stoianovitch3 who advocated surgical repair, based on accumulated cases from the literature. Surgery became the accepted treatment of Achilles tendon rupture. This period was characterized by statement such as "Patients with a rupture of the Achilles tendon should be operated on and should be operated on without delay. 1

Hippocrates view of rupture of the calcaneal tendon was that this tendon, if bruised or cut, causes the most acute fevers, induces choking, deranges the mind and at length brings death. 4

In 2002, in a systemic review and meta-analysis, Bhandari et al.5 pooled the results of 448 patients eandomized to either operative or nonoperative treatment following Achilles tendon rupture and reported a relative risk of rerupture of $0.32(95 \%$ confidence interval $(\mathrm{Cl}), 0.14$ to 0.71 ) in favor of surgical repair. A regimen for functional treatment after repair of the Achilles tendon was first described by Marti and Weber.6 Those authors removed the plaster cast four, five or six days and instructed the patients in active range of motion exercise for the ankle and knee. After motion of the ankle had almost returned to normal, a below the knee plaster cast was worn for six to eight weeks and the patient was allowed progressive weight bearing. Since the late 1980s some new regimens involving early motion have been reported.7 All of these regimens had satisfactory results without an increase in the rate of failure or a substantial elongation of the tendon. 8

Recent reports in the literature have favored operative treatment of an acute rupture of the Achilles tendon. In a retrospective study of 314 patients who had undergone open repair between 1980 and 1991, Winter et al.9 noted that nine patients had delayed wound healing, ten had deep wound infection requiring additional operative treatment and two had a sinus necessitating debridement and closure. In the report by Cetti et al.10 on open repair in fifty-six of 111 patients, $4 \%$ had deep wound infection, $2 \%$ had delayed healing, $10 \%$ had adhesion of scar tissue and 12\% had disturbance of sensation. although Nystrom and Holmlund11 reported that seperation of the AT ends followed a biphasic course, with an initial separation of the AT ends followed a biphasic course, with an initial separation in the interval 0 to 7 days, no seperation in 8 to 12 days and late separation in 22 to 35 days, the present elongation curves first rose and then slowly fell in both groups. After 6 weeks, the AT preserved its length or even shortened a little in early motion group.

Cetti et al.12 showed that patients treated postoperatively for 6 weeks with a mobile cast were able to resume sports activities sooner than those treated for the same length of time with a below knee cast with the ankle in a 20 degree eqine position for 6 weeks. Rantanen et al.13 reported good results obtained by Achiles rupture treatment using early postoperative immobilization of the ankle in a neutral position, but no controlled comparisons with other regimens have been reported. Since the late 1980s, there has been a trend toward functional postoperative treatment, which has been reported to be well tolerated, safe and effective in compliant, well motivated patient. In a metaanalysis by Khan et al.14 the rerupture rates were estimated at $3.5 \%$ and $12.6 \%$ in surgically and non-surgically treated patients, respectively. Other authors have pointed out that surgical treatment is associated with a higher rate of other complications, such as infections, wound problems, nerve injuries and adhesion after surgery. Early weight bearing and mobilization with or without surgical treatment is suggested to produce the best result, provided that the tendons end are in contact. 15

Tendoachilles injury is the 3rd most common tendon injury. Primary surgical treatment with restoration of normal anatomy in a single operation is required to achieve the best possible outcome. If possible, specially with sharp injuries, it is better to repair severed tendons initially than to delay because in secondary repair it requires tendon graft, a lengthy multidisciplinary voyage. The functional outcome of a Achilles tendon injury after a repair depends on multiple factors such as age, injury level and type, type of repair as well as post repair care. Scarring, adhesion formation and subsequent stiffness have been the major hindrance to good results after a Achilles tendon repair. In our study we will try to evaluate the outcomes of primary repair of acute Achilles tendon injuries of leg by means of regular follow up of the patient till 16 weeks of repair which will encourage primary repair where possible

\section{BEFORE WE START}

\subsection{Anatomy of Tendo Achilles}

The tendinous portions of the Gastronemius and Soleus muscles merge to form Achilles Tendon. Over $90 \%$ cases Plantaris muscle is present medial to the tendon. The Gastrocnemius tendon originates as a broad aponeurosis at the distal margin of muscle bellies, whereas the Soleus tendon begins as a band proximally on the posterior surface of the soleus muscle. Distally the tendon become progressively rounded in cross section, to a level $4 \mathrm{~cm}$ proximal to calcaneal tuberosity, where it can become relatively flatter before inserting on the superior aspect of calcanean tuberosity. The fibers of the Achillies tendon spiral through 90 degrees during its descent, such that fibers that lies medially in the proximal portion become posterior distally. In this way elongation and elastic recoil within the tendon are possible and stored energy can be released during the appropriate phase of locomotion.15 Also this stored energy allows the generation of higher shortening velocity greater instantaneous muscle power than could be achieved by contraction of Triceps surae alone. The calcaneal insertion of the Achilles tendon is highly specialized, as it is composed of attachment of the tendon, a layer of hyalin cartilage and an area of bone not cover by periosteum. A subcutaneous bursa may lie between the tendon and the skin 
to reduce friction between the tendon and the surounding tissues. A retro calcanean bursa lies between the tendon and the calcaneus.

\subsection{Structure of the Tendon}

Tendons act as transducers of the force produced by muscle contraction to bone. Collagen accounts for 70 percent of the dry weight of a tendon. Apprximately 95 percent of tendon collagen is type-1 collagen, with a very small amount of elastin. Elastin can undergo as much as 200 percent strain before failure. If it were present in the tendon in high proportions, there would be a decrease in the magnitude of force transmitted to bone. Collagen fibrils are bundled into fascicles containing blood and lymphatic vessels as well as nerves. The fascicles are grouped together, surrounded by epitenon and form the gross structure of the tendon, which is further enclosed by paratenon, seperated from the epitenon by a thin layer of fluid to allow tendon movement with reduced friction. Although the normal Achilles tendon also contsists almost entirely of type-1 collagen, a ruptured Achilles tendon also contains a substantial proportion of type-3 collagen. Fibroblast from ruptured Achilles tendon produce both type -1 and type- 3 collagen on culture.

Type- 3 collagen is less resistant to tensile forces and may therefore predispose the tendon to spontaneous rupture. The normal Achilles tendon shows a well organized cellular arrangement in stark contrast to one that is ruptured.16 Tenocytes, which are specialized fibroblast, appear in transverse sections as stellate cells and are arranged in rows in longitudinal sections. This orderly arrangement probably is due to the uniform centrifugal secretion of collagen around the column of tenocytes, which produce both the fibrillar and non-fibrillar components of the extracellular matrix and may also reabsorb the collagen fibers.

\section{$>$ Blood Supply-}

Tendons can receive their blood supply from vessels originating from three sources: the musculo tendinous junction, the surrounding connective tissue and the bone tendon junction. The blood flow of the Achilles tendon depends on age, with a higher blood flow in younger individuals. The Achilles tendon is poorly vascularized, especially in its midportion, with blood vessels running from the paratenon into its substance. There is a dispute concerning the distribution of blood vessels in the tendon. Some investigations have shown that the density of blood vessels in the middle part of the Achilles tendon is low compared with that in the proximal part. Others have shown, with use of laser Doppler flowmetry that blood flow is evenly distributed throuhout the Achilles tendon and may vary according to age, gender and loading conditions. 17

\section{$>$ Biomechanics of the Tendon-}

Actin and myosin are present in tenocytes and the tendon itself may have an active contraction-relaxation mechanism, which could regulate the transmission of force from muscle to bone. Fukashiro et al.18 measured a peak force of 2233 newtons within the human Achilles tendon in vivo. Komi et al.19 used buckle-type force transducers attached to the ankles of volunters to show that, during walking, force builds up within the tendon before the heel strikes the ground. The force is then suddenly released for ten to twenty milliseconds during early impact. There after force builds up relatively fast until it reaches a peak at the end of the push-off phase, in a pattern similar to that observed during running. More recently, Arndt et al.20 showed that the Achilles tendon can be subjected to non uniform stresses through modifications of individual muscle contributions. An injury therefore can be produced by a discrepancy in individual muscle forces caused, for example, by asynchronous contraction of the various components of the triceps surae or by uncoordinated agonist-antagonist muscle contraction due to impaired transmission of peripheral sensory stimuli. At rest, a tendon has a wavy configuration, accounting for the toe-region of the stress starin curve. As collagen fibers deform, they respond linearly to increasing tendon loads. If the strain placed on the tendon remains at less than 4 percent, that is within the limits of most physiological load. The fibers regain their original configuration on removal of the load. At strain levels between 4 and 8 percent, the collagen fibers start to slide past one another as the intermolecular cross links fail. At strain levels of greater than 8 percent, macroscopic rupture occurs because of the tensile failure of the fibers and inter fibrillar shear failure. The compliance of the tendon is dependent at least in part on intratendinous waviness, which may affect the ability of the gastrocnemiussoleus muscle complex to generate force at the extrems of joint motion.21 Ultimately, it may also influence the forces exterted by muscle contraction on the tendon and hence the propensity.

\section{$>$ Healing Mechanism of tendon-}

During tendon healing fibroblastic infiltration from the adjacent tissue essential. The healing events are initiated by an inflammmatory response, which is characterized by polymorphonuclear cell infiltration, capillary budding and fluid exudation and which continue during the first three days following injury. After four days fibroplasias occurs and is accompanies by the significant accumulation of fibroblasts. Within three weeks, a mass of granulation tissues surrounds the damaged tissue. During the next week, collagen fiber become longitudinally oriented. During the next three months, the individual collagen fibers form bundles identical to the original buldles. Sutured tendons heal with a progressive penetration of connective tissue from the outside. The deposited collagen fibers become progressively oriented untill eventually they form tendon fibers like the original ones. The orientation of collagen fiber is essential because the tensile strength of repaired tendon is dependent on collagen content and orientation. If tendon is sutured the first 7-10 days of healing, the strength of the suture maintains the fixation untill adequate callus has been formed.

Tendon mobilization during heading is important to avoid adhesion of the tendon to the adjacent tissue, particularly in case involving flexors tendons of hand. Motions can be passive to prevent adhesions and at the same 
time to prevent putting excessive tensile stress on the suture line.

\section{$>$ Mechanism of Injury-}

Open tendo Achilles injury has been found to be caused by various mechanism. Commonly it is inflicted by different types of sharp cutting weapons such as knife during assault or as a part of domestic accident fall in the toilet pan etc.

Most commonly the mechanism of rupture are pushing off with the weight bearing fore foot while extending the knee, sudden unexpected dorsiflexion of the ankle and violent dorsiflexion of the planter flexed foot as in all form height. Disruption can also occur from a direct blow to the contracted tendon or from a laceration.

\section{Different modalities of treatment-}

The advantage of surgical treatment of Achilles tendon rupture iies in the possibility of exerting early tension on the tendon which will ensure appropriate orientation of collagen fibers and improve the strength of the calf muscle. While an increasing number of recent reports in the literature have tended to favor operative treatment of an acute rupture of the Achilles tendon, the exact type of operative procedure as well as post-operative regiment remains controversial. Surgical repair of Achilles tendon rupture is associated with improved calf strength on cybex testing and reduced tendon lengthening and may have a smaller rerupture rate than nonoperative management.22 There is no single, uniformly accepted surgical technique and surgical options include open repair with or without augmentation and per cutaneous technique. In Europe many authorities use strong monofilament or braided absorbable sutures, whereas in North America the tendency is to use mechanically strong stiches extending well above and below the site of rupture.23 Authors supporting operative intervention have suggested that the normal tensin and length of the tendon can be restored only under direct visualization during an open repair.24 On the basis of many recent reports in the literature we believe that rupture of the Achilles tendon are best treated operatively and we were attracted to the method described by Kakiuchi, which combines the advantage of open and percutaneous technique.

\section{METHODOLOGY}

This was a prospective cohort study done in the Department of Orthopaedices, Rajshahi Medical College Hospital in between 1st January, 2017 to 31st December, 2017. Sampling technique was purposive sampling. For estimating sample size, the following formula was used: $n$ $=\mathrm{Z} 2 \mathrm{pq} / \mathrm{e} 2$, where,

$\mathrm{p}=$ prevalence or proportion of occurrence; if not known, it is regarded as $0.5(50 \%)$,

$q=1-p, Z=1.96$; at $5 \%$ level of significance or $95 \%$ confidence level,

$\mathrm{e}=0.05$ (10\% of $\mathrm{p})$; acceptable error or precision in the estimate of ' $p$ '.
For this study, the sample size was calculated to be $384(p=0.5 \%)$. Since, 384 is too big sample size for a study period of one year, a sample size of 50 was deemed to be acceptable.

\section{Selection criteria:}

$\checkmark$ Inclusion criteria:

Patients with the following conditions were included-

- Age: Patients of 12- 60 years of both sexes.

- Achilles tendon injuries of both legs with minimal tissue loss.

- Incised and incised looking wound within 12 hours of injury.

- Achilles tendon injuries of leg, skin were closed primarily but tendon was not repaired (within 10 days of injury).

\section{$\checkmark$ Exclusion criteria:}

Patients with the following conditions were excluded-

- Crush injuries with huge soft tissue loss, burn and charring.

- Associated fracture and neurovascular injuries.

- Poly- trauma

- Patient suffering from unstable medical illness.

\section{Operational definitions}

Zones of Achilles tendon of Leg: Tear at the musculotendinous junction treated conservatively using a plantar flexed cast or boot for 6-9 weeks. Mid-substance tendon tears 2 to $6 \mathrm{~cm}$ above the tendon insertion into the calcaneous can be treated by surgical repair.33

1. Primary repair of tendons: Repair within 24 hours of injury.

2. Delayed primary repair: Repair after 24 hours to approximately 10 days of injury.

Surgical technique: Admission of the patient under Department of Casualty Surgery $\rightarrow$ Assessment $\rightarrow$ Operation (repair of the tendon by Modified Kesslar Method using Prolene 1 with round bodied needle and paratendon by using Vicryl 4-0 with round bodied needle, skin with 3-0 proline $\mathrm{C} / \mathrm{B}$ by using regional anesthesia and tourniquette control $\rightarrow$ Short leg anterior slab $>$ Follow up.

\section{$>$ Procedure of data analysis:}

Structured questionnaire and data sheet were used to collect data. Results were compiled manually by classical tally method in broad sheets. Classification and summation of data were made. Results were put in tables, charts and bars and discussed accordingly. Data was processed and analyzed using computer software SPSS (Statistical Package for Social Sciences). The test statistics to be used are descriptive statistics, Chi-square $(\chi 2)$ and Student's t-Test. Level of significance was set at 0.05 and pa $<0.05$ was considered significant. 


\section{$>$ Quality control measures:}

A good quality of data collection was ensured .For that purpose at first, sample size was selected and a standard data sheet was made. Then the data sheet was pretested. The pretest ensured that the data were valuable regarding the study.

\section{> Ethical implications:}

- Participation was voluntary.

- Written informed consent was obtained from either the patient or attendant after explaining the aims \& objectives of the study, along with the message that, they are free to take part or refuse to be enrolled in this study.

- Collected information was kept confidential. The procedure was helpful for both the physicians and the patients in making rational approach of the case management.

- Prior to commencement of this study, the research protocol has been submitted to the ethical committee of Dhaka Medical College. Ethical Clearance Certificate was issued by Chairman, Ethical Review Committee, Dhaka Medical College, Dhaka for the study on 16.02.2014 and the memo no: DMC$\mathrm{MEU} / \mathrm{ECC} / 2014 / 43$.

\section{$>$ Preoperative evaluation}

- Detail history is taken

- Clinical examination done methodically.

- Documentation of clinical examination

- Pre-operative evaluation for fitness for surgery is evaluated both clinically and by lab investigations.

- X-ray of local part-Anterior, Posterior and Lateral View.

\section{$>$ Operative technique-}

After proper preoperative counseling and discussing with the patient or patients guardian about the merits and demerits of the operative procedure, informed written consent was taken. After admission tetanus prophylaxis was given to all patients. Oral cefuroxime $250 \mathrm{mg} 12$ hourly and flucloxacillin 500mg 6 hourly was given for 2 weeks. If needed antibiotic changes according to culture sensitivity report. The operation performs under local anesthesia in a prone position. Then through surgical toileting was done with normal saline and soap. Aseptic surgical toileting was done with a mixture of Hxiscrub, povidone iodine, hydrogen peroxide and normal saline. Finally wound was washed with 4 to 5 liter normal saline and a swab was taken for culture and sensitivity test. After surgical toileting tourniquet was applied in the thigh, then wound painting with antiseptic solution especially with povidoniodine and proper drapping was done. A posteromedial skin incision was made and the fascia and paratenon was divided in the same line. The tendon was repaired by modified Kessler suture technique with nonabsorable number 1 proline. Paratenon was repaired by vicryl. Skin closure was done with proline. Optimal operative outcome demands good operative technique, respect for contraindications and appropriate patient selection. After repairing the tendon short leg anterior slab was kept with knee at 40 degree flexion and ankle at 10 degree flexion in gravity equinus position. Isometric quadriceps exercise, toe movement was advised. Patients were discharged 1st postoperative day and were advised to walk with a pair of crutch without weight bearing.

\section{Postoperative follow up}

All of the patients were requested to come to the hospital for regular follow up according to following protocol. Follow up ranges from 6 months to 12 months. In every follow up visits the patient was asked for any symptoms, looked for sign of infection and after plasters removal examined clinically to assess power of planter flexion, calf muscle wasting, ability of stand on affected limb and range of movement of ankle of affected limb. Patient was come for follow up on 3rd postoperative days and examine for any sign of infection. Patient was advised to continue quadriceps and hamstring strengthening exercise and walk with the aid of pair of crutch. Patients were come for 2 nd visit at 14 th pod. Stiches removal was done and below knee anterior slab changed to below knee plaster cast with ankle at gravity equinus position for 4 weeks. Patients were advised for quadriceps strengthening exercise, knee movement, hip movement within the active range of motion. Patients were also advice for non weight bearing walking with crutches. When any infection presents swab was taken for culture and antibiotic changes according to culture sensitivity Patient was advice to do regular dressing until infection subsides. Patients were come for 3 rd visit at 6 th weeks. At that time plaster was removed and below knee plaster cast given with ankle in neutral position for another 4 weeks. Patients was advice for continue exercise and non weight bearing walking with crutches. Then after 10 weeks plaster removal was done when patients come for 4th visit. Patient was advised for walking with 2 inches heel raised shoe and with crutch for further 2 to 4 weeks. Manual therapy like soft tissue mobilization to decrease oedema, cross friction massage to scar, soft tissue mobilization technique to planter fascia and flexior halluces longus, ankle mobilization to subtalar joint and talocrural joint was advised. Therapeutic quadriceps strengthening exercise, knee movement, ankle tubing exercise for ankle joint dorsiflexion, planter flexion, inversion and eversion was advised. Patients also advice for towel curls, towel sweep for non-weight bearing to partial weight bearing at affected foot as tolerated along with standing bilateral heel raise exercise. Patients were advice not to do jumping or running. Patients were come for 5 th visit at 12 th weeks. Then patients were advised for full weight bearing with affected limb without help of walking aid. Patients was advised to do manual therapy along with therapeutic exercise like standing calf stretching, balance exercise, gluteal heep knee strengthing exercise with swiss ball and side stepping exercise with elastic tubing along with standing single leg heel rise exercise. Patient was come for final visit at 16th weeks. At that time final outcome assessment of the patient as done according to Juhana leppilahti scoring system.25,26 Subsequent visits were advised 3 months interval till the satisfactory functional improvement of injured leg achieved. Jumping, running or heavy weight lifting was allowed after 6 months. 
Assessment of outcome after Primary Repair According to Juhana Leppilahti scoring systemAt least 90 points- Excellent-outcome

75 to 89 points- Good-outcome

60 to74 points - Fair-outcome

Less than 60 points - Poor outcome

\begin{tabular}{|c|c|c|c|c|c|c|}
\hline & \multicolumn{4}{|c|}{ Sex } & \multicolumn{2}{c|}{$\begin{array}{c}\text { Mean } \\
\pm \text { SD }\end{array}$} \\
\cline { 2 - 7 } & \multicolumn{2}{|c|}{ Male } & \multicolumn{2}{c|}{ Female } & Percentage & \\
\hline $12-25$ & 8 & Percentage & Number & Percentage & $100 \%$ & $30.02 \pm 7.71$ \\
\hline $26-35$ & 20 & 76.0 & 4 & 34.0 & $100 \%$ & $100 \%$ \\
\hline $36-45$ & 10 & 83.0 & 2 & 33.0 & $\mathbf{1 0 0 \%}$ & \\
\hline Total & $\mathbf{3 8}$ & $\mathbf{7 6 \%}$ & $\mathbf{1 2}$ & $\mathbf{2 4 \%}$ & \\
\hline
\end{tabular}

Table 3.1 Age distribution of the study patients according to $\operatorname{sex}(n=50)$

Among 50 patients $38(76.0 \%)$ were male and $12(24.0 \%)$ were female. Mean age was 30.02 years with standard deviation of \pm 7.71 .

\begin{tabular}{|c|c|c|}
\hline Causes of injury & Number of patients & Percentage \\
\hline Broken toilet pan & 25 & 50.0 \\
\hline Sharp weapon & 15 & 30.0 \\
\hline Machinery & 10 & 20.0 \\
\hline
\end{tabular}

Table 3.2 Distribution of the study patients by causes of injury $(\mathrm{n}=50)$

Table 3.2 shows $50 \%$ injury was due to sharp edge of broken toilet pan following slip on Indian type of toilet pan.

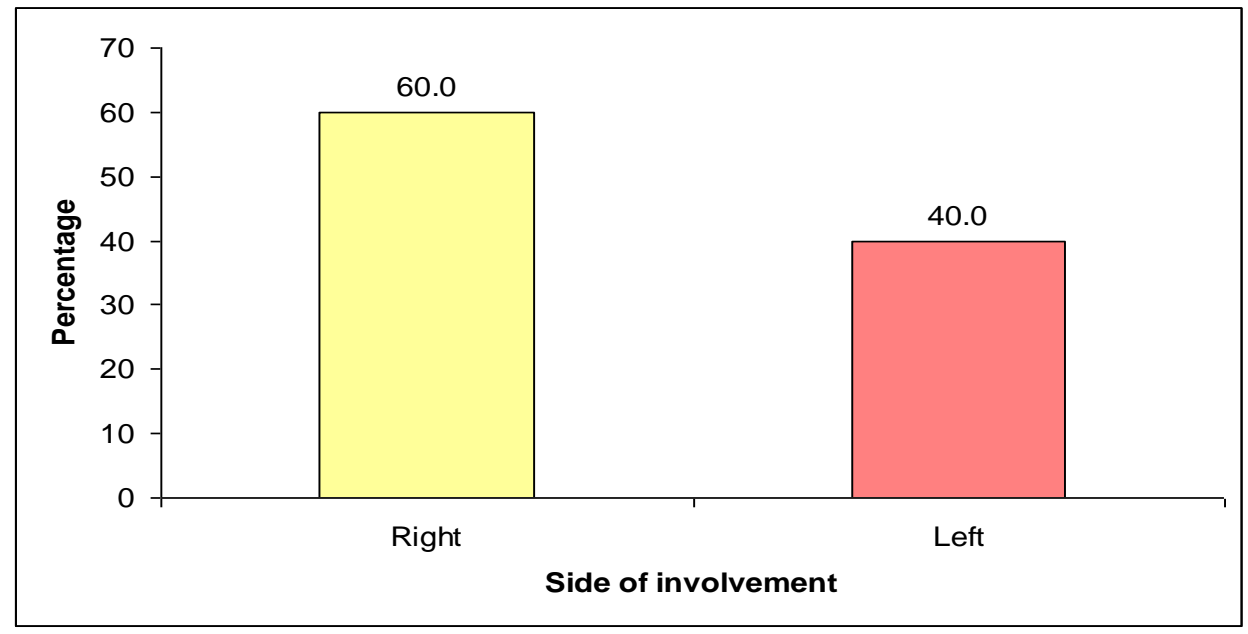

Figure 3.1 Bar diagram showing side of involvement of the study patients $(n=50)$

Figure 3.1 showsmore affected involvement was right side 30(60\%) and left side 20(40\%).

\begin{tabular}{|c|c|c|c|c|}
\hline Level of cut & Number & Percentage & $\begin{array}{l}\text { Median Level of } \\
\text { cut }(\mathrm{cm})\end{array}$ & Interquartile range(IQR) \\
\hline $2-3 \mathrm{~cm}$ & 25 & 50.0 & \multirow{4}{*}{3.0} & \multirow{4}{*}{2.5 to 3.5} \\
\hline $3-4 \mathrm{~cm}$ & 19 & 38.0 & & \\
\hline $4-5 \mathrm{~cm}$ & 5 & 10.0 & & \\
\hline$>5 \mathrm{~cm}$ & 1 & 2.0 & & \\
\hline Total & 50 & 100.0 & & \\
\hline
\end{tabular}

Table 3.3 Distribution of the study patients by level of cut due to acute injury $(n=50)$ 
In this series median level of cut was $3.0 \mathrm{~cm}$ where interquartile range was 2.5 to $3.5 \mathrm{~cm}$.

\begin{tabular}{|c|c|c|c|c|}
\hline Delay & Number & Percentage & Mean delay & Standard deviation \\
\hline$<4$ hours & 4 & 8.0 & \multirow{4}{*}{$\begin{array}{l}6 \text { hours } \\
\text { minutes }\end{array}$} & \multirow{4}{*}{ \pm 2.362} \\
\hline 4-6 hours & 30 & 60.0 & & \\
\hline 6-8 hours & 8 & 16.0 & & \\
\hline 10-12 hours & 8 & 16.0 & & \\
\hline Total & 50 & 100.0 & & \\
\hline
\end{tabular}

Table 3.4: Distribution of the study patients by treatment delay $(n=50)$

All the cases were operated on emergency basis after admission through emergency department. Most of the cases were operated within 6 hours of injury.

Mean delay of repair was 6 hours 7 minutes, with standard deviation \pm 2.362 minutes.

\begin{tabular}{|c|c|c|c|c|c|}
\hline $\begin{array}{c}\text { Follow up } \\
\text { period in } \\
\text { month }\end{array}$ & Frequency & $\begin{array}{c}\text { Minimum Period } \\
\text { (In month) }\end{array}$ & $\begin{array}{c}\text { Maximum Period } \\
\text { (In month) }\end{array}$ & $\begin{array}{c}\text { Mean follow } \\
\text { up period } \\
\text { (In month) }\end{array}$ & $\begin{array}{c}\text { Standard } \\
\text { deviation }\end{array}$ \\
\hline $5-8$ & 20 & 5 & 16 & 9 \\
\hline $9-12$ & 27 & 5 & 5.37 & \\
\hline$>12$ & $\mathbf{5 0}$ & & & & \\
\hline Total & & & & \\
\hline
\end{tabular}

Table 3.5 Distribution of the study patients by showing total follow up period after surgery $(\mathrm{n}=50)$

All the patients advised for regular follow up at Orthopaedics out door or in Orthopaedics indoor of RMCH. One or two visits for initial two weeks then regular visits at four weeks interval upto total return of normal day work. Minimum and maximum follow up period was 5 and 16 months respectively. Mean follow up period was 9 months with standard deviation was 2.37 .

\begin{tabular}{|l|l|l|}
\hline Swab culture sensitivity & Number of patients & Percentage \\
\hline E. Coli & 30 & 60.0 \\
\hline Pseudomonas & 3 & 6.0 \\
\hline Klebshiela & 2 & 4.0 \\
\hline No growth & 15 & 30.0 \\
\hline
\end{tabular}

Table 3.6 shows after final toileting wound swab was sent for culture sensitivity and found in $60.0 \%$ cases E.Coli, $6.0 \%$ Pseudomonas, $4.0 \%$ Klebshiela and in $30.0 \%$ cases no growth.

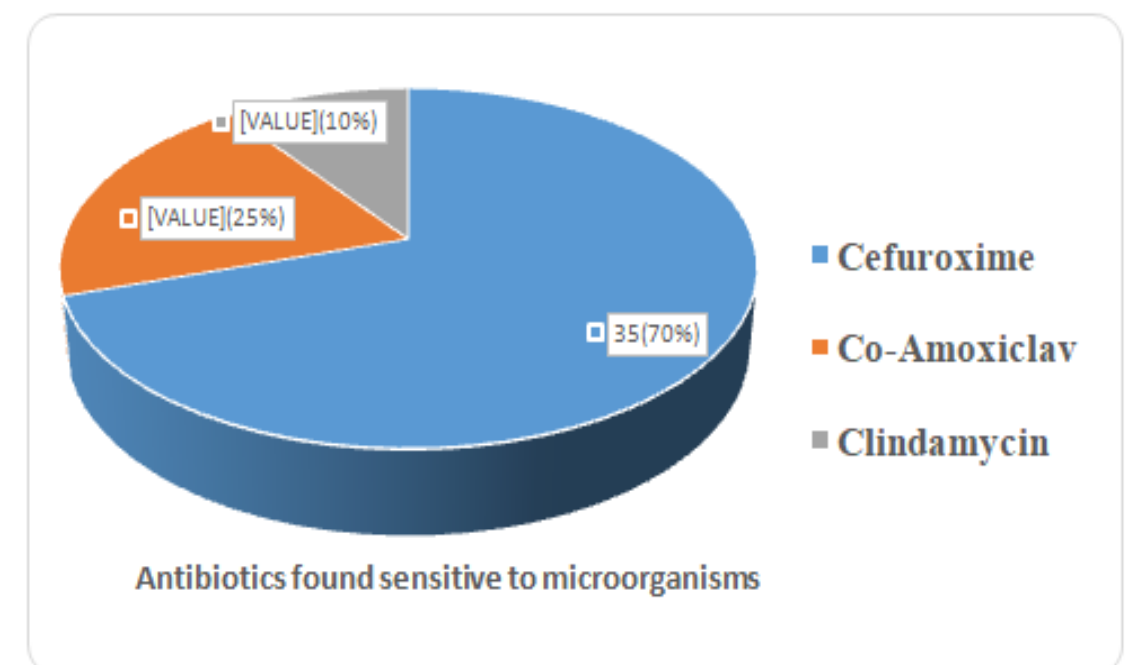

Figure 3.2Pie chart showing different antibiotics found sensitive to microorganisms 
Figure 3.2 shows in $70 \%$ cases I had not to change my prescribed antibiotics at first time, because of $70 \%$ organism were sensitive to Cefuroxime others changed changed accordingly.

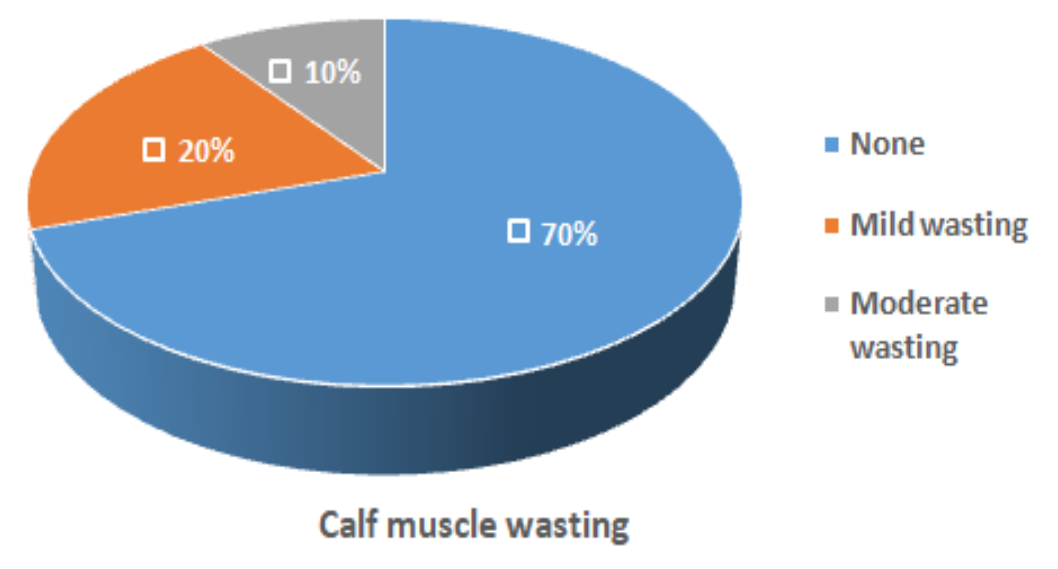

Figure 3.3Pie chart showing calf muscle wasting of the study patients $(\mathbf{n}=\mathbf{5 0})$ side.

Figure 3.3 shows calf muscle wasting measured as maximum calf circumference as a ratio between injured and non-injured

Table 3.7 Distribution of the study patients by power plan flexion (BMRC) $(\mathrm{n}=50)$

\begin{tabular}{|l|l|l|}
\hline Power plan flexion (BMRC) & Frequency & Percentage \\
\hline Power 2 & 1 & 2.0 \\
\hline Power 3 & 4 & 8.0 \\
\hline Power 4 & 10 & 20.0 \\
\hline Power 5 & 35 & 70.0 \\
\hline Total & $\mathbf{5 0}$ & $\mathbf{1 0 0 . 0}$ \\
\hline
\end{tabular}

Table 3.7 shows majority $35(70.0 \%)$ patients had power plan flexion was 5 followed by $10(20.0 \%)$ was 4 , 4(8.0\%) was 3 and1 $(2.0 \%)$ was 2 .

Table 3.8 Distribution of the study patients by range of motion of ankle joint $(n=50)$

\begin{tabular}{|l|l|l|}
\hline Range of motion of ankle joint & Frequency & Percentage \\
\hline Normal & 35 & 70.0 \\
\hline Mildly limited & 13 & 26.0 \\
\hline Moderate limited & 2 & 4.0 \\
\hline Total & $\mathbf{5 0}$ & $\mathbf{1 0 0 . 0}$ \\
\hline
\end{tabular}

Table 3.8 shows majority $35(70.0 \%$ ) patients had normal range of ankle joint, $13(26.0 \%)$ mildly and 2(4.0\%) moderate range of ankle joint. 
Table 3.9 Distribution of the study patients by ability to stand on affected tip toes or foot $(\mathrm{n}=50)$

\begin{tabular}{|l|l|l|}
\hline Ability to stand on affected tip toes or foot & Frequency & Percentage \\
\hline Can stand on affected tip toes & 45 & 90.0 \\
\hline Cannot stand on affected toes but stand on & 4 & 8.0 \\
\hline Cannot stand on affected foot unsupported & 1 & 2.0 \\
\hline Total & $\mathbf{5 0}$ & $\mathbf{1 0 0 . 0}$ \\
\hline
\end{tabular}

Table 3.9 shows majority $45(90.0 \%)$ patients were can stand on affected tip toes, $4(8.0 \%)$ were cannot stand on affected toes but stand on and only $1(2.0 \%)$ cannot stand on affected foot unsupported.

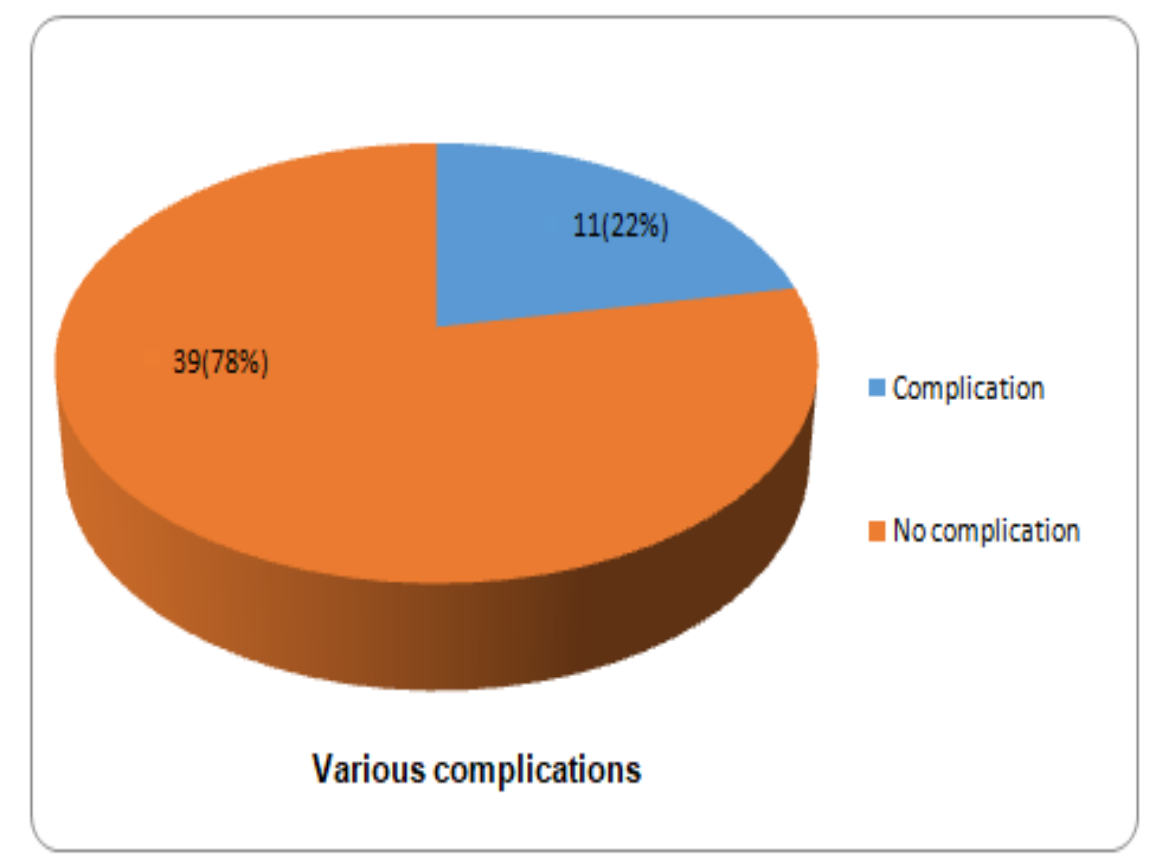

Figure 3.4 Pie chart showing various complications of the study patients $(n=50)$

Figure 3.4 shows $39(78 \%)$ cases found no complications and another 11(22\%) cases found different types of complications.

Table 3.10 Distribution of the patients by various minor complications $(n=11)$

\begin{tabular}{|l|l|l|}
\hline Minor complications & Number of patients & Percentage \\
\hline Superficial skin infection & 4 & 36.4 \\
\hline Mild Swelling & 4 & 36.4 \\
\hline Ugly scar & 2 & 18.2 \\
\hline Wound gap & 1 & 9.1 \\
\hline
\end{tabular}

Table 3.10 shows regarding minor complications, 4(36.4\%) patients had superficial skin infection, 4(36.4\%) had mild swelling, 2(18.2\%) had ugly scar and $1(9.1 \%)$ had wound gap. 
Table 3.11 Comparison of Range of motion of ankle joints (Planter flexion and Dorsi flexion) between healthy and injured sides after treatment

\begin{tabular}{|c|c|c|c|}
\hline \multirow[t]{2}{*}{ Outcome } & Injured limb & Healthy limb & \multirow[t]{2}{*}{ Pvalue } \\
\hline & Mean \pm SD & $\operatorname{Mean} \pm$ SD & \\
\hline $\begin{array}{l}\text { Range of motion of ankle joint (Planter } \\
\text { Flexion) }\end{array}$ & $36.52 \pm 2.76$ & $39.8 \pm 0.61$ & $\mathrm{P}>0.05$ \\
\hline $\begin{array}{l}\text { Range of motion of ankle joint (Dorsi } \\
\text { Flexion) }\end{array}$ & $22.02 \pm 3.11$ & $24.9 \pm 0.30$ & $\mathrm{P}>0.05$ \\
\hline
\end{tabular}

Range of motion of ankle joints (Planter flexion and Dorsiflexion) of injured and healthy sides were measured at the last follow up. As sample size was greater than 30, so one sample z-test was done for calculation of test statistics and we found that there were no significant differences between range of motion of ankle joints (Planter flexion and Dorsi flexion) of injured and healthy sides.

Table 3.12 Distribution of the patients by final outcome $(\mathbf{n}=50)$

\begin{tabular}{|l|l|l|}
\hline Grade & Number of patients & Percentage \\
\hline Excellent & 28 & 56.0 \\
\hline Good & 16 & 32.0 \\
\hline Fair & 4 & 8.0 \\
\hline Poor & 2 & 4.0 \\
\hline Total & $\mathbf{5 0}$ & $\mathbf{1 0 0 . 0}$ \\
\hline
\end{tabular}

Satisfactory $=$ Excellent + Good

$$
\begin{aligned}
& =56 \%+32 \% \\
& =88 \%
\end{aligned}
$$

Unsatisfactory $=$ Fair + Poor

$$
\begin{aligned}
& =8 \%+4 \% \\
& =12 \%
\end{aligned}
$$

\section{TEST OF SIGNIFICANCE - CONFIDENCE INTERVAL}

In this study it was found that $85 \%$ satisfactory result among 50 patients of acute open tendo Achilles injured patients by immediate repair. If this procedure put in total population then satisfactory result was found in following confidence interval (at $5 \%$ level of significance).

Confidence interval $(\mathrm{CI})=p \pm 1.96 \sqrt{\frac{p q}{n}}$ (at $5 \%$ level of significance $)$

$$
\text { Here, } \begin{aligned}
\mathrm{p} & =88 \\
\mathrm{q} & =(100-\mathrm{p}) \\
& =12 \\
\mathrm{n} & =50
\end{aligned}
$$

So, $\mathrm{CI}=88 \pm 1.96 \sqrt{\frac{88 * 12}{50}}$

$$
\begin{aligned}
=88 \pm & 1.96 \times 4.596 \\
& =88 \pm 9.0075 \\
& =78.99 \% \text { to } 97.00 \%
\end{aligned}
$$

According to Juhana Leppilahti Modified Scoreat 95\% Confidence Interval satisfactory outcome $=78.99 \%$ to $97.00 \%$ So, early repair of open Tendo Achilles injury provided an effective outcome 


\section{DISCUSSION}

Rupture of the Achilles tendon was reportedly first described by Ambroise Pare in 1575 and was reported in the literature in 1633. After the 1920s the use of operative treatment increased, primarily because of reports by Abrahamsen in 1923 and Quenu and Stoianovitch in 1929 who advocated surgical repair, based on accumulated cases from the literature. 1

The primary outcome was the rerupture rate. Rerupture was diagnosed by the investigating surgeon on the basis of a positive Thompson squeeze test, the presence of a palpable gap and loss of plantar flexion strength. Secondary outcome included isokinetic strength, the leppilahti score, ankle range of motion and calf circumference. A meta analysis showed the rate of rerupture to be approximately $2.5 \%$ following operative repair of an Achilles tendon rupture and $13 \%$ following nonoperative treatment. 27

Beskin et al.28 showed that the surgically treated Patients had increased strength and a decreased rerupture rate. In a randomized prospective study, Cetti et al.10 concluded that operative treatment of a ruptured Achilles tendon is preferable, but non-operative treatment is an acceptable alternative. From the literature review of operative treatment (4477 patients), the rerupture rate was $1.5 \%$ as compared with the non-operative casting group (569 patients), where the rerupture rate was $13.3 \%$. It is clearly demonstrated that the chances of rerupture are considerably decreased after operative intervention.

Both surgical and non-surgical therapies are followed by immobilization in a cast or movable brace. Meta-analysis generally agree that the rerupture rate is higher in patients treated non-surgically (12.6) than in patients treated surgically $(3.5 \%)$. Furthermore, regardless of treatment type, patients often have residual weakness and decreased function after Achilles tendon rupture.49 patients were treated surgically. Post operatively the patients were placed in a below the knee cast with the foot in 30 degree equines position.48 patients composed the non-surgical group. The patients were treated immediately with a below the knee cast with the foot in equuinus position. There were no significant difference between the groups with regard to the patient's own opinions about their symptoms and function or their physical activity levels at the 6 and 12 months evaluations. The surgical group had significantly better results in the heel rise work, heel rise height, concentric power and hopping tests at the 6 months evaluation than did the nonsurgical group. 29

Surgical repair of acute Achilles tendon rupture is associated with improved calf strength on cybex testing and reduced tendon lengthening and may have a smaller rerupture rate than non-operative management. The $1-5 \%$ incidence of delayed wound healing and $0.4-2 \%$ incidence of wound breakdown and necrosis following open repair may be reduced by using a percutaneous technique. 30
While an increasing number of recent reports in the literature have tended to favor operative treatment of an acute rupture of the achilles tendon, the exact type of operative procedure as well as the postoperative regimen remains controversial. Many surgeons favor a formal operative approach to secure the best possible repair with the least chance of rerupture. Our standard treatment of an acute rupture of the Achilles tendon has long been an open operative approach followed by cast immobilization for eight weeks. However we have noted a disturbing rate of problems with wound healing and infection. In addition, some of our patients were dissatisfied with the unsighty and occasionally painful scars. 31

Until the 1980s, casting was the standard postoperative regimen after Achilles tendon rupture, regardless of the surgical technique used. The ankle was conventionally immobilized in an equinus position without weight bearing for 4 to 9 weeks. All the patients were managed with the same operative technique. One author repaired 42 of the ruptures and six other surgeons operated on the remaining 8 . The operations were performed under spinal anesthesia in a prone position using a tourniquet. A posteromedial skin incision was made and the fascia and paratenon were divided in the same line. The tendon was repaired by the two modified Kessler suture technique with absorable polydiaxone 2-0 sutures and smaller apposition sutures with vicryl. After suturing, the ankle was easily placed in a neutral position. The fascia was carefully resutured with Vicryl and the skin was closed with Ethilon sutures. At the end of the operation, a below knee rigid plaster splint was applied with the ankle in a neutral position. 32

There is no consensus on the best treatment for acute Achilles tendon ruptures. Although non-operative treatment has its own supporters, surgical treatment seems to be the method of choice in the case of athletes and young people and for delayed ruptures. The outcome was assessed at the 3 month and final checkups by the clinical scoring method described by Kangas et al.25. The scoring included subjective factors such as pain, stiffness, muscle weakness, footwear restrictions and subjective outcome and objective factors such as the range of active ankle motion and isokinetic calf muscle strength.

In Europe, many authorities use strong monofilament or braided absorbable sutures, whereas in North America the tendency is to use mechanically strong stiches extending well above and below the site of rupture with braided nonabsorbable material. The present ankle performance scores at the last control visit were excellent or good in $88 \%$ of the case in the early mention group, fair in $4 \%$ and poor in $8 \%$, whereas the scores in the cast group were excellent or group in $92 \%$ and fair in $8 \%$.Thus no significant differences were detected between the groups at either the 3 months or the final check-up. The performance scores in both groups were better than in the series of Kangas et al.32, in which the postoperative treatment consisted of 6 weeks of below knee cast immobilization with the ankle in an equinus position for 3 weeks and in a neutral position for 3 weeks, allowing gradual weight bearing after 3 weeks, for which the scores 
were excellent or good in $79 \%$ of the 101 cases, fair in $17 \%$ and poor in $4 \%$ at a mean of 3 years postoperatively. Part of the reason that our results were better might be the homogenous patient groups, because patients over 60 years, patients with systemic diseases and patients with late Achilles tendon ruptures were excluded in the present study.

This study is a prospective cohort, done with 50 cases of tendo achilles rupture treated by modified Kessler method of operative technique. This period was characterized by statement such as "Patients with a rupture of the Achilles tendon should be operated on and should be operated on without delay.1 After the 1920s the use of operative treatment increased, primarily because of reports by Abrahamsen2 and Quenu and Stoianovitch3 who advocated surgical repair, based on accumulated cases from the literature. Surgery became the accepted treatment of Achilles tendon rupture. Rupture of the Achilles tendon was reportedly first described by Ambroise Pare in 1575 and was reported in the literature in 1633.34

Commonly it is inflicted by different types of sharp cutting weapons such as knife, during assault or as a part of domestic accident by a kitchen knife or during road traffic accident or by accidental cut by broken glass or by accidental fall in the toilet pan etc. 1 In 2002, in a systemic review and meta-analysis, Bhandari et al.5 pooled the results of 448 patients randomized to either operative or nonoperative treatment following Achilles tendon rupture and reported a relative risk of rerupture of 0.32 (95\% confidence interval $(\mathrm{Cl}), 0.14$ to 0.71 ) in favor of surgical repair rupture of the Achilles tendon is one of the most common tendon injuries in the adult population. The incidence of this injury is increasing as aging adults continue their participation in high demand sports. 27 In our study 25 cases (50\% injury) were caused by sharp edge of broken toilet pan following slip on Indian type of toilet pan, 15 cases (30\%) were caused by sharp weapons and machinery injury was occurred for around 10 cases $(20 \%)$.

In a study Carden et al.4 found rupture of the Achilles tendon is more common in males with a female ratio ranging from 1.7:1 to $12: 1$. Saw et al.7 consisted that there were 19 patients, 16 men and 3 women, with a mean age of 37.7 years (range 25-60 years). Another study conducted by Suchak et al.26 where they reported that mean age of the patients was $40 \pm 15$ years. Of the 315 patients, $84 \%$ were men. In our study among 50 patients $38(76 \%$ ) were male and $12(24 \%)$ were female. Mean age was 30.02 years with standard deviation of \pm 7.71 .

Primary repair of tendons means repair within 24 hours of injury. In Europe many authorities use strong monofilament or braided absorbable sutures8, whereas in North America the tendency is to use mechanically strong stitches extending well above and below the site of rupture. 8 Authors supporting operative intervention have suggested that the normal tension and length of the tendon can be restored only under direct visualization during an open repair.24 All the tendons were repaired by Modified Kesslers method with non absorbable suture. A postero- medial skin incision was made and the fascia and paratenon was divided in the same line. The tendon was repaired by modified Kessler suture technique with nonabsorable number 1 proline. Paratenon was repaired by vicryl. Skin closure was done with proline.25 Many surgeons favor a formal operative approach to secure the best possible repair with the least chance of rerupture.35 Optimal operative outcome demands good operative technique, respect for contraindications and appropriate patient selection.36 In our study all the cases were operated on emergency basis after admission through emergency department. Most of the cases were operated within 6 hours of injury. After final toileting wound swab was sent for culture sensitivity and found in $60 \%$ cases E. Coli, 6\% Pseudomonas, 4\% Klebshiela and in $30 \%$ cases no growth. In $70 \%$ cases I had not to change my prescribed antibiotics at first time, because of $70 \%$ organism were sensitive to Cefuroxime axetil others changed accordingly.

The peak incidence of Achilles tendon ruptures occurs in the middle age group rather than in the older population suggested a mechanical etiology for tendon rupture.1 Mean delay of repair was 6 hours 7 minutes, with standard deviation \pm 2.362 minutes. Lagergren and Lindholm37 noted that ruptures of the Achilles tendon are usually limited to a definite segment of the tendon 2 to $6 \mathrm{~cm}$ proximal to its insertion on the calcaneus. In our study median level of cut was $3.0 \mathrm{~cm}$ proximal to the insertion of Tendo Achilles and where interquartile range was 2.5 to $3.5 \mathrm{~cm}$. It was found excellent or good results those cut occurred within $3-4 \mathrm{~cm}$ proximal from the insertion.

Saw et al.7 reported that all patients were assessed between 5 months and 2 years after operation, with a mean of 13.6 months. Chatterjee et al.38 published their study of acute Tendo Achilles injury of 18 patients. The follow up of the 15 patients ranged from 5 months to 10 years. In our study all the patients advised for regular follow up at Orthopaedics out door or in Orthopaedics indoor of RMCH. One or two visits for initial two weeks then regular visits at four weeks interval upto total return of normal day work. Minimum and maximum follow up period was 5 and 16 months respectively. Mean follow up period was 9 months with standard deviation was 2.37 .

Reduction of calf circumference is usual, even many years after rupture. In this study it was found reduction of calf circumference was as little as described by other.39

Mandelbaum et al.23 reported that the patients was instructed to move the ankle passively as comfort allowed four to five times a day through $10^{\circ}$ to $20^{\circ}$ of both plantar flexion and dorsiflexion. If the wound had fully healed, early weight bearing, coupled with range of motion exercises, was initiated. Patients were fitted with hinged walking boots that permitted full plantar flexion but blocked dorsiflexion at $10^{\circ}$. Changes the motion of the ankle, indicates, tendon shortening or lengthening.40 Ankle motion were normal in 35 cases $(70 \%)$ and changes of motion were up to 6-10 degree in $13(26 \%)$ patients in this series, which was very much acceptable in comparison to 
above studies. Only 2(4\%) patient had more than 10 degree motion restricted.

The power of planter flexion was normal or near normal (90\%) 40 cases. Various types of complications were encountered among the patients of this study. In 39(78\%) cases found no complications and another 11(22\%) cases found different types of complications. Common complications were superficial skin infection 4 cases (37\%), mild swelling 4 cases (36\%), ugly scar 2 cases (18\%) and wound gap 1 case $(9 \%)$. The causes of Achilles tendon elongation may be numerous. Technical causes can include failure of the suture material or slipping of the knot or necrosis around the sutures that allows one of them to cut through the tendon.25 A rapid return to normal mobility with normal plantarflexion strength was found. Patients regained mobility significantly quicker, and were able to return to normal activities sooner. No patient had excessive lengthening of the tendon. The incidence of tendocutaneous adhesion (two out of 19 patients, 10.5 percent) seems to be less than that reported in the literature. Lea and Smith41 and Nistor42 reported their incidence of tendocutaneous adhesion to be 15 percent and 45 percent, respectively. Other potential complications of prolonged plaster immobilization can also be avoided. Normal muscle strength is readily attained, compared with published results, whereby only approximately 83 per cent of plantarflexion strength was achieved at long-term follow-up.42,43 The slight loss of the range of plantarflexion (mean= 5.6") in their study did not compromise the end result.

Early repair of open Tendo Achilles injury provided an effective outcome, which was comparable with the result described in others. 44

Saw et al.7 found that out of 19 patients, 15 patients were asymptomatic and had no complications. There were two patients with minor wound infection at 2 weeks which settled on antibiotics with no long-term sequelae. One of these two patients had a slightly decreased sensation of the lateral skin edge. Two other patients had tendocutaneous adhesions. There were no ruptures. Post operative infection after operative treatment for Achilles tendon ruptures have been reported with a range of 4-20\%.29 Moller et al.40 and Nistor42 reported more adhesions, disturbed sensibility, and deep or superficial wound infection in the operative group. No statistical analysis was performed. In our study no major complication occurred but minor complications like superficial skin infection, delayed wound healing occurred which were successfully managed with an oral antibiotic regimen with no long term sequelae. One patient with wound gap had to readmit in hospital for sural island flap. All patients returned to their normal activities after a variable period of 6 to 16 months without complaints. There was no rerupture case in this study.

In this series we used 5 parameters according to Juhana Leppilahti Modified scoring system to assess the final outcome of the patient functionally. These were stand on affected tip toe unsupported, range of motion of affected side ankle, power of planter flexion, calf muscle wasting and complications. These parameters were also used in various study described in various literature.40,45 The patients were asked about their sporting activities before and after treatment and whether they had any complaints, they were also asked to evaluate their own results as excellent, good, fair or poor. In this series we assessed fifty acute open Tendo Achilles injured patients after 16 weeks of treatment. Among them 90\%(45) can stand on affected tip toes unsupported, around 4 cases( $8 \%)$ cannot stand on tip toes of affected foot unsupported but able to stand on affected foot unsupported, around 2\% (1 case) failed to stand on affected foot unsupported due to severe infection and wound gap.

The final result was as followed excellent in 28 cases (56\%), good in 16 cases (32\%), fair in 4 cases $(8 \%)$ and poor in 2 cases (4\%).

In this study it was found that $85 \%$ satisfactory result among 50 patients of acute open tendo Achilles injured patients by immediate repair. If this procedure put in total population then satisfactory result was found in following confidence interval (at $5 \%$ level of significance). Calder and Saxby30 consisted that the mean Leppilahti score was 86 at 3 months (range 60-90) with 12 patients scoring excellent, 28 good, and 6 fair. At 6 months the mean Leppilahti score was 96 (range 82-100) with 42 patients scoring excellent and 4 scoring good. All patients stated they were satisfied with their functional and cosmetic results According to Juhana Leppilahti Modified Score at 95\% Confidence Interval satisfactory outcome is $78.99 \%$ to $97.00 \%$, which was comparable with the result described in others. So, early repair of open Tendo Achilles injury provided an effective outcome, which was comparable with the result described in others. 44

\section{LIMITATIONS OF THE STUDY}

This study lacks some strengthening factors. One is the sample size which could be a bigger one in other set up except a prerequisite for fellowship training. Sampling technique was purposive sampling. A non-purposive one with a bigger sample size could also be helpful to determine the types and nature of fistula in our country.

Our study is a prospective cohort study which lacked adequate power to determine differences in patient preoperative variables such as demographic factors. True comparison and advantage of primary repair of injured tendo achilles may not be clear until larger prospective, randomized studies are performed.

Only patients with tendo achilles injuries were in the study. With experience and success of primary repair an attempt may later be taken into consideration to include complicated injuries in further study. Moreover open repair of tendon by modified kessler is old method compare to percutaneous repair technique. 
USG or MRI were avoided to reduce treatment cost but it should be included in the study to get a clear preoperative idea about the achilles tendon.

\section{CONCLUSION}

With the current reported data in consideration, we believe that primary repair procedure of injured tendo achilles is a safe and more economical technique with minimal tissue injury and low recurrence rates with well monitored post operative follow up shedules. This technique of repair with well monitored follow up encouraging early results with good outcomes. The technique of primary repair of injured tendo achilles is promising and has potential to be a valid option with good post operative outcome following physiotheraphy. Attention to details of the technique with regular follow up is the key to success regarding outcome variables. But only then it could be said as the best technique with good outcomes and low complications when it is compared with modern s techniques.

\section{RECOMMENDATIONS}

True of primary repair of injured tendo achilles may not be clear until larger prospective, randomized studies are performed. It could be compared with newer techniques like percutaneous repair procedure as an alternative to the previously well-established open modified kessler procedure. Randomized controlled trials with long-term follow-up are necessary to include primary repair of injured tendo ahilles as a standard treatment option.

USG or MRI investigating tools on further study may be considered later regarding the effectiveness of procedure Table 2. Tables may span across both columns

\begin{tabular}{|l|l|l|l|l|}
\hline Column1 & Column2 & Column3 & Column4 & Column5 \\
\hline Cell 1 1 & Cell 1 2 & Cell 1 3 & Cell 1 4 & Cell 1 5 \\
\hline Cell 2 1 & Cell 2 2 & Cell 2 3 & Cell 2 4 & Cell 2 5 \\
\hline Cell 3 1 & Cell 3 2 & Cell 3 3 & Cell 3 4 & Cell 3 5 \\
\hline Cell 4 1 & Cell 4 2 & Cell 4 3 & Cell 4 4 & Cell 4 5 \\
\hline
\end{tabular}

+ The example for this table. The example for this table. The example for this table. The example for this table. The example for this table. The example for this table..

If the square-shaped pixel size in our images was $8 \times 8$ screen-pixels, this amounted to about 21 pixels per face quantization (an equivalent of about 10.5 cycles/face). With this level of image detail, all three basic varieties of configural information (hange of spatial quantization between 11 pixels/face and 6 pixels/face levels altogether indicate that this ERP- component is especially sensitive to the first-order configural cues. Some other works have supported both of these ideas $[6,16,25]$.

\section{ACKNOWLEDGMENTS}

We acknowledge our heartiest gratitude to all doctors and colleagues in the department of Surgery, Rajshahi Medical College \& Hospital, Rajshahi, for their inspiration, co-operation and valuable suggestions.
We might be failing in our sense of gratitude unless we offer our gratefulness to those patients who so optimistically offered them to be a subject in our research.

\section{REFERENCES}

[1]. Popovic N, Lemaire R. Diagnosis and Treatment of Acute Ruptures of The Achilles Tendon Current Concepts Review. Acta Orthopaedica Belgica 1999; 65(4):458-471.

[2]. Abrahamsen H. Ruptura tendinis achillis. Ugeskr. Laeg 1923; 85:279-284.

[3]. Quenu J, Stoianovitch SM. Les ruptures du tendon d'Achille. Rev. Chir 1929; 48:647-678.

[4]. Carden DG, Noble J, Chalmers J, Lunn P, Ellis J. Rupture of the calcaneal tendon: the early and late management. J Bone Joint Surg 1987; 69-B:416-20.

[5]. Bhandari M, Guyatt GH, Siddiqui F, Morrow F, Busse J, Leighton RK, Sprague S, Schemitsch EH. Treatment of acute Achilles tendon ruptures: a systematic overview and metaanalysis. Clin Orthop Relat Res 2002; 400:190-200.

[6]. Marti RK, Weber BG. Rupture of the achilles tendon functional after care. Helv Chir Acta 1974; 41:293296.

[7]. Saw Y, Baltzopoulos V, Lim A, Rostron PK, BoltonMaggs BG, Calver RF. Early mobilization after operative repair of ruptured Achilles tendon. Injury 1993; 24: 479-484.

[8]. Mortensen NHM, Skov O, Jensen PE, Denmark O. Early Motion of the Ankle After Operative Treatment of a Rupture of the Achilles Tendon. The Journal of Bone and Joint Surgery 1999; 81-A(7):983-989.

[9]. Winter E, Weise K, Weller S, Ambacher T. Surgical repair of Achilles tendon rupture. Archives of Orthopaedic and Trauma Surgery 1998; 117: 364-367.

[10]. Cetti R, Christensen SE, Ejsted R. Operative versus nonoperative treatment of Achilles tendon rupture: A prospective randomized study and review of the literature. Am J Sport Med 1993; 21:791-9.

[11]. Nystrom B, Holmlund D. Separation of tendon ends after suture of Achilles tendon. Acta Orthop Scand $1983 ; 54: 620-621$.

[12]. Cetti R, Henriksen LO, Jacobsen KS. A new treatment of ruptured Achilles tendons. Clin Orthop 1994; 308:155-165.

[13]. Rantanen J, Hurme T, Paananen M. Immobilization in neutral versus equinus position after Achilles tendon repair. Acta Orthop Scand 1993; 64:333-335.

[14]. Khan RJ, Fick D, Keogh A, Crawford J, Brammar T, Parker M. Treatment of acute achilles tendon ruptures: a meta-analysis of randomized, controlled trials. J Bone Joint Surg Am 2005; 87(10):2202-2210.

[15]. Maffulli N, Longo UG, Denaro V. Complications after surgery or nonoperative treatment for acute achilles tendon rupture. Clin J Sport Med 2009; 19:441-442.

[16]. Strocchi R, De Pasquale V, Guizzardi S, Govoni P, Facchini A, Raspanti M, Girolami M, Giannini S. Human Achilles tendon: morphological and 
morphometric variations as a function of age. Foot and Ankle 1991; 12: 100-104, 1991.

[17]. Astrom M, Westlin N. Blood flow in the human Achilles tendon assessed by laser Doppler flowmetry. J. Orthop. Res 1994; 12:246-252.

[18]. Fukashiro S, Komi PV, Jarvinen M, Miyashita M. In vivo Achilles tendon loading during jumping in humans. European J. Appl. Physiol. and Occup. Physiol 1995; 71: 453-458.

[19]. Komi PV, Fukashiro S, Jarvinen M. Biomechanical loading of Achilles tendon during normal locomotion. Clin. Sports Med 1992; 11:521-531.

[20]. Arndt AN, Komi PV, Bruggeman GP, Lukkariniemi B. Individual muscle contribution to the in vivo Achilles tendon force. Clin Biomech 1998; 13:532541.

[21]. Hawkins D, Bey M. Muscle and tendon force-length properties and their interactions in vivo. J. Biomech 1997; 30:63-70.

[22]. Lo IK, Kirley A, Nonweiler B. Operative versus nonoperative treatment of acute Achilles tendon ruptures: a quantitative review. Clin J Sports Med 1997; 7:20711.

[23]. Mandelbaum BR, Myerson MS, Forster R. Achilles tendon ruptures: a new method of repair, early range of motion and functional rehabilitation. Am J Sports Med 1995; 23:392-395.

[24]. Bradley JP, Tibone JE. Percutaneous and open surgical repairs of Achilles tendon ruptures. A comparative study. Am J Sports Med 1990;18:188-95.

[25]. Kangas J , Pajala A, Ohtonen P, Leppilahti J. Achilles Tendon Elongation After Rupture Repair-A randomized Comparison of 2 postoperative Regimens. American Journal of Sports Medicine 2007;35(1):5964.

[26]. Suchak AA, Spooner C, Reid D. Postoperative Rehabilitation Protocols for Achilles Tendon Ruptures. Clinical Orthopaedics and Related Research 2006; 45:216-21.

[27]. Willits K, Amendola A, Bryant D, Mohtadi NG, Giffin JR, Fowler P. Operative Versus Nonoperative Treatment of Acute Achilles Tendon Ruptures: A Multicenter Randomized Trial Using Accelerated Functional Rehabilitation. The American Journal of Bone and Joint Surgery 2010; 92:1-9

[28]. Beskin, JL, Sanders, RA, Hunter SC. Surgical repair of Achilles tendon ruptures. Am J Sport Med 1987; 15: 18.

[29]. Helander KN, Silbernagel KG, Thomee R, Faxen E. Acute Achilles Tendon Rupture-A Randomozed Controlled Study Comparing Surgical and Nonsurgical Treatment Using Validated Outcome Measures. The American Journal of Sports Medicine 2010; 20(10):18.

[30]. Calder JDF, Saxby TS. Early active rehabilitation following mini-open repair of Achilles tendon rupture: a prospective study 2005; 39:857-859.

[31]. Assal M, Jung M, Stern R, Rippstein P. Limited Open Repair of Achilles Tendon Ruptures. The Journal of Bone and Joint Surgery 2002; 84-A(2):161-169.
[32]. Kangas J, Pajala A, Sirra P, Hamalainen M. Early Functional Treatment Versus Early Immobilization in Tension of the Musculotendinous Unit after Achilles Rupture Repair: A Prospective, Randomozed, Clinical study. The Journal of Trauma injury, Infection and Critical Care 2003; 54(6):1171-118.

[33]. Williams N, O'Connell PR, McCaskie A. Bailey \& Love's Short Practice of Surgery, 27th edition, CRC Press, Published March 21, 2018.

[34]. Pare A. Les Oeuvres, 9th ed. Lyon; Claude Rigaud et Vlaude Obert, 1633,

[35]. Wallace RGH, Traynor IER, Kernohan WG, Eames MHA. Combined Conservative and Orthotic Management of Acute Ruptures of the Achilles Tendon. The Journal of Bone and Joint Surgery America 2004;86-A(6):1198-1202.

[36]. Azar FM, Canale ST, Beaty JH. Campbell's Operative Orthopaedics, 4 volume set. 13th edition, Orthopedic surgery $2017 ; 1-4746$.

[37]. Lagergren C, Lindholm A. Vascular distribution in the Achilles tendon: An angiographic and microangiographic study. Acta chir. scan dinav 1959; 116:1958.

[38]. Chatterjee SS, Sarkar A, Misra A. Management of acute open tendo-achilles injuries in Indian lavatory pans. Indian J Plast Surg 2006; 39(1): 29-33.

[39]. Wagdy-Mahmoud S, Megahed AH, El-Sheshtawy O. Repair of the calaneal tendon. I Bone Joint Surg 1992; 74: 114-117.

[40]. Moller M, Movin T, Granhed H, Lind K, Fraxen E, Karlsson J. Acute rupture of tendo achillis. A prospective randomized study of comparison between surgical and nonsurgical treatment. Journal of bone and joint surgery $2001 ; 83: 843-848$.

[41]. Lea RB, Smith L. Non-surgical treatment of tendo Achilles rupture. J. Bone joint Surg 1972; 54A: 1398.

[42]. Nistor L. Surgical and non-surgical treatment of Achilles tendon rupture. J. Bone joint Strrg 1981; 63A: 394.

[43]. Shields C, Kerlan R, Jobe F. The Cybex II evaluation of surgically repaired Achilles tendon ruptures. Am. J. Sports Med 1978; 6: 369.

[44]. Strauss EJ, Ishak C, Jazrawi L, Sherman O, Rosen J. Operative treatment of acute Achilles tendon ruptures: an institutional review of clinical outcomes. Injury 2007; 38: 832-838.

[45]. Aldam CH. Repair of the calcaneal tendon. A safe technique 1989; 71-B(3):486-88. 